\title{
"La Fuite et la recherche de soi et d'un nouveau monde chez Le Clézio"
}

Par

\section{Noha Abdel Aziz Rizq*}

noha.mabrouk@art.aun.edu.eg

\section{Résumé}

Le thème de la fuite acquiert une grande importance et occupe une place primordiale dans les cuvres de le Clézio dès son premier roman. Dans tous ses romans et surtout "le Livre des fuites" où il ne fait qu'exprimer une angoissante inquiétude de vivre en harmonie dans ce monde moderne. Sa vie et son œuvre se balancent entre deux pôles extrêmes : l'univers urbain et la vie citadine d'un côté et par la vie des éléments de la nature d'autre côté. Mais il veut toujours confirmer que la vie matérielle a changé la belle image de la terre et même, elle a corrompu les valeurs humaines. Selon lui, le langage qui domine la vie moderne n'est plus le langage de la communauté puisqu'il est fondé sur l'intérêt particulier, l'utilitarisme, la domination et même sur l'esclavage. C'est la cause pour laquelle il annonce sa méfiance et son inquiétude. "Le Livre des fuites" ne fait qu'accentue et accélère la montée de la violence, de la solitude, de la peur et par conséquence la fuite qui consistue l'objet de cette étude où cette fuite a deux aspects essentiels : la fuite du monde matériel, et la recherche de soi et d'un nouveau monde.

\footnotetext{
* Professeur adjoint, Département de Français,Faculté des Lettres, Université d'Assiout
} 


\section{Introduction}

Jean-Marie Gustave Le Clézio, le lauréat du prix de Renaudot en 1963 et du prix Nobel de littérature en 2008, est un des écrivains les plus célèbres de la littérature française et francophone du $X X^{\mathrm{e}}$ siècle. Il a eu cette célébrité grâce à ses idées, son style, et son originalité qui vient de sa recherche perpétuelle à être le soi dans toutes ses écritures, de sa singulière maturité dans la maîtrise de l'écriture, et de sa conscience aiguë.

Le thème de la fuite acquiert une grande importance et occupe une place primordiale dans les œuvres de Le Clézio dès son premier roman : Le Procès-Verbal publié en 1963 passant par La Fièvre (1965), Le Déluge (1966), L'Extase matérielle(1967), $\underline{\text { Terra amata (1967), Le Livre des Fuites (1969), La guerre }}$ (1970), Haï (1971) et Les géants (1975). La Fuite est le "thème récurrent à l'obsession, thème pourtant inépuisable ${ }^{\prime 1}$ dans tous ses romans et surtout Le Livre des fuites où il ne fait qu'exprimer une angoissante inquiétude de vivre en harmonie dans ce monde moderne au point qu'il veut :

"Fuir, toujours fuir. Partir, quitter ce lieu, ce temps, cette peau, cette pensée. M'extraire du monde abandonner mes propriétés, rejeter mes mots et mes idées et m'en aller"'? 
On doit savoir que dès le début de sa carrière littéraire Le Clézio décrit le monde urbain, le monde mécanique et même l'agression du langage et ses formes. Mais quelle est l'origine de sa pensée à ce propos ?

En effet, et d'après ses œuvres, nous trouvons que sa vie et son œuvre se balancent entre deux pôles extrêmes : Le Clézio luimême avoue qu'il a été tenté par l'univers urbain et la vie citadine d'un côté et par la vie des éléments de la nature d'autre côté. Le Clézio voulait confirmer que la vie moderne et toutes ses valeurs matérielles ont changé la belle image de la terre et même, elles ont corrompu les valeurs humaines :

"dans les grandes villes (on ne trouve que) "la méchanceté, la cruauté, la déshumanisation ...etc. (...) (la ville moderne est) l'espace d'abus, de violence, de famine, de haine, de désespoir en bref de toutes sortes de mal" ${ }^{3}$.

Il assure sa pensée dans $\underline{\text { Terra amata }}$ lorsqu'il dit :

"Il y avait la méchanceté des hommes (...),

la voracité des animaux, et l'indifférence des objets(...) II $\mathbf{y}$ avait tous ces bruits, toutes ces lumières, toutes ces odeurs, comme autant de coups de poignard qui frapperaient tout le temps dans la chai ${ }^{\prime 4}$. 
Comme on a marqué, il y a constamment cette insistance sur le thème de la fuite et la peur de vivre.

Car il s'incline "à être "soi" tant dans le domaine littéraire que dans sa vie personnelle. Cette vocation de l'écrivain à se focaliser sur "soi" tout en cherchant à s'identifier à ce qui existe dans le monde ${ }^{\prime \prime}$. Et Le Clézio voit toujours que le monde urbain et la vie mécanique conjurent son angoisse et exorcisent sa panique. C'est ainsi que la ville moderne, d'après lui, au lieu de protéger l'homme et de lui offrir un refuge, multiplie, au contraire, les agressions et les dangers :

"La ville se traduit comme un symbole de cruauté, d'agressi vité et de solitude"6.

En tant qu'observateur, le danger se trouve partout. Béton, asphalte, métal, électricité, gaz, atome, plastique et plusieurs autres objets menacent la vie quotidienne. Toutes ces matières ont effacé dans le corps du monde de la ville, sa belle image :

"Les progrès techniques et la civilisation n'ont fait qu'aggraver les risques de la situation de la vie humaine, au lieu de réaliser ses rêves"

D'autre part, le bruit représente un élément premier de cette pollution qui agresse, à la fois, les sens et les nerfs. En effet, Le Clézio exprime la peur de vivre dans un style scientifique, 
rapide, tendu, nerveux et harcelé pour concrétiser le spectacle de la folie et l'enfer de la ville.

Mais à quoi aspire Le Clézio ? il aspire à un langage silencieux entre l'homme et les choses puisque "le silence est vraiment célébré comme une vertu cardinale dans toute l'auvre le clézienne" ${ }^{18}$.

Il est convaincu que le langage qui domine la vie moderne n'est plus le langage de la communauté puisqu'il est fondé sur l'intérêt particulier, le mensonge, l'utilitarisme, la domination et même sur l'esclavage. C'est la cause pour laquelle Le Clézio annonce sa méfiance et son inquiétude. Des œuvres comme Le Livre des

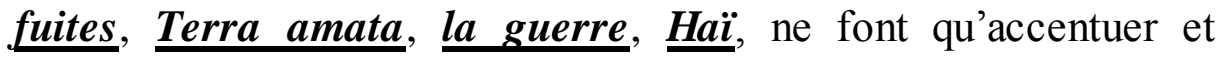
accélérer la montée de la violence, de la solitude, de la peur et par conséquent la fuite qui constitue l'objet de cette étude. Cette fuite a, dans les œuvres de notre corpus, deux aspects essentiels : la fuite, et la recherche de soi et d'un nouveau monde :

\section{- La Fuite :}

Les personnages le cléziens se comportent et vivent comme des étrangers dans le monde terrestre et éprouvent un sentiment d'isolement. Dans Le livre des fuites, on découvre que le personnage principal est un jeune homme qui parcourt le monde moderne et les villes ne trouvant nulle part que la peur de vivre et l'insécurité. Le Clézio appelle cette œuvre le livre des fuites un 
roman d'aventures. Cependant, il s'en échappe à toutes les formes et les genres conventionnels conçus par les critiques. Le titre même de cette œuvre romanesque de Le Clézio est significatif. Car il n'est pas question d'une fuite précise, d'un monde moderne, mais fuite des formes, fuite de soi et même fuite de la fuite elle-même :

"Le titre suggère d'entrée de jeu le caractère négatif de l'entreprise du personnage, ou du moins une certaine urgence sous-entendue par le titre au pluriel "fuites"".

Ce que Le Clézio trace dans son roman est d'une nature fictive, puisqu'il affirme au début de son roman que c'est lui qui fait les routes et les chemins :

"Je regarde tous ces pays qui sont à moi, tous les fleuves qui coulent pour moi, dit-il. Je regarde ce masque peint qui est le visage de ma terre. Je prends possession, comme du haut d'une tour. Je suis partout chez moi" ${ }^{10}$.

C'est ainsi que le but principal de ce livre ne se rattache ni au divertissement ni à ce qu'on pouvait appeler l'exotisme. Le Clézio affirme à ce propos que : 
"l'exotisme est un vice, parce que c'est une manière d'oublier le but véritable de toute recherche, la conscience $^{\prime \prime 11}$.

Par conséquent, le but dans le livre des fuites ne désigne pas un déplacement géographique, mais simplement une fuite imaginaire et une plongée dans la conscience :

"Je veux n'être qu'un avec la force qui me pousse, ditil. Je suis un wagon. Sous mes routes, les rails froids sont étendus, que j’aime un éclair de ma chaleur qui dévale le bruit sinistre de l'air. Ce tourbillon est ma pensée. Je suis un naufragé de la terre" ${ }^{12}$.

Cependant, Le Clézio souligne dans Le Livre des fuites que la fuite des lieux, des gens et de la ville est inutile, car les chaînes et les limites se trouvent partout :

"Quitter pour quoi, pour qui ? trouver un autre monde, habiter une autre ville (...) Vivre sous un autre ciel ? Non pas cela, je ne veux pas mentir. Les chaînes sont partout (...) Ce n'est pas cela qu'il faut quitter. Un déplacement géographique vers, un petit glissement vers la droite, ou vers la gauche, à quoi bon ? "13 
Hagon, héros du livre des fuites s'évade sans aucune raison plausible, mais simplement pour mieux connaître le spectacle du monde moderne : ses mécanismes et sa réalité :

"Terre, je te fuis, c'est pour mieux te connaître"14.

Remarquons que le romancier emploie plusieurs formes d'écriture: chiffres, noms, devinettes, insultes, critique, autocritique et aussi la poésie :

"loin de la méchanceté

loin très loin

loin du vice, du malheur, de la haine, qu'on m'emporte loin.

Très loin.

par les navires

par les avions de fer

par les routes de tonnerre

au pays du loin

du grand, du brûlant, du vibrant

du lointain loin"15.

D'autre part, pour fuir, c'est d'abord se fuir :

"quitter ce lieu, ce temps, cette peau, cette pensée (...) fuir la fuite même, nier jusqu'à l'ultime plaisir de la négation. Entrer en soi, se dissoudre, s'évaporer sous le 
feu de la conscience, se résoudre en cendres, vivement, sans répit" ${ }^{16}$.

Fuir signifie donc une fuite plus interne qu'externe, une descente et une aventure dans des zones inconnues de la psyché :

"Ce qui demeure paradoxal dans la fuite du protagoniste, c'est qu'elle ne s'assigne pas comme objectif la découverte ou l'exploration exotique d'un pays déterminé. La fugue, avec ses aléas et ses revirements, est une tentative de se connaître, d'entrer en communion avec sa propre conscience" ${ }^{\prime 17}$.

En outre, Le Clézio à travers la fuite "aspire à une nouvelle forme de conscience, à une nouvelle vision "18.

Dans Le livre des fuites, Le Clézio emploie et développe un ensemble de disparition et d'effacement, d'affirmation et de négation. Dans cette optique, la pensée de Le Clézio se caractérise par une vision qui repose sur une infinité de moyens où chaque affirmation comporte sa propre négation :

"le blanc est noir, dit-il"19.

Et dans un autre passage, il écrit:

"Je veux tracer ma route, puis la détruire, ainsi, sans repos. Je veux rompre ce que j’ai

(La Fuite et la recherche de soi...) Dr. Noha Abdel Aziz Rizq 
créé, pour créer d'autres choses, pour les rompre encore. C'est ce mouvement qui est le vrai mouvement de la vie : créer, et rompre. Je veux imaginer pour effacer l'image" ${ }^{20}$.

C'est ainsi que se fonde la pensée de Le Clézio : l'ordre et le désordre, l'unité et la pluralité dans un mouvement qui s'ouvre sur l'infini :

"Quand je suis un (dit-il), je suis tous. J'ai l'ordre, aussi, le contre-ordre, de rompre ma rupture dès qu'elle est advenue. Il n'y a pas de vérité possible, mais pas de doute non plus (...) Je veux être mouvement, mouvement qui va, qui n'avance pas, qui ne fait qu'énumérer les bornes. Une frontière s'ouvre, c'est une nouvelle frontière qui apparaît (...) Je dis femme, c'est-à-dire statue, c'est-à-dire pieuvre, c'est-àdire roue (...) Yin, c'est-à-dire Yang" ${ }^{21}$.

Dans "The French Review", Cagnon, Maurice et Smith, Stephen disent dans l'article "Le Clézio Taoïste vision" que l'ancienne philosophie chinoise de la cosmologie est basée sur deux principes fondamentaux desquels se dérivent toutes les pensées réelles et imaginaires. Yang, le principe ou le pôle actif masculin est associé physiquement avec la chaleur, le feu, la lumière, l'aridité et toutes ses dérivées imaginaires et symboliques

(La Fuite et la recherche de soi...) Dr. Noha Abdel Aziz Rizq 
qui représentent ainsi la vie active. Yin, le pôle féminin, le principe passif est la source des ombres, la profondeur, l'humidité, la froideur qui représentent la vie négative ou la mort. Selon les philosophes Taoïste ${ }^{22}$, Yang et Yin renferment dans leur polarité toutes les oppositions fondamentales de l'existence. La relation de Yang-Yin permet d'expliquer l'œuvre, la philosophie et la créativité imaginaire dans l'œuvre de Le Clézio qui emploie les deux principes de vie et de mort, d'affirmation et de négation comme autant des métaphores privilégiées à toute expression et à toute métamorphose. Il parle également de lui-même ou de ses personnages fictifs en autant des récepteurs passifs des forces venues du monde extérieur.

Pour représenter la violence du monde moderne, Le Clézio se sert d'un certain nombre de mots tranchants comme rasoirs, lames, couteaux, harpons, clous, comme des clés de comparaison.

En effet, Le Clézio parle de l'invention humaine des armes, des machines et des outils meurtriers. Le narrateur de $\underline{\text { Terra Amata }}$ énumère les diverses images de la mort, simplement pour montrer le plaisir sadique chez l'homme :

"La violence sordide est partout étalée. Il y a partout des mains aux ongles durs qui étranglent et qui mutilent. Les yeux regardent avidement le spectacle 
brûlant et glacial, et le monde n'est que nourriture. Le crime approche ... il arrive" ${ }^{23}$.

Chez Le Clézio, on peut remarquer facilement cette pulsion de vie et de mort qui prédomine et oriente les multiples images dans l'œuvre de cet écrivain. Ses personnages s'exposent et s'ouvrent au monde comme des récepteurs passifs à toutes les forces venues du monde extérieur :

"On en est farcis tous de sensations"

Dans un autre endroit, nous lisons :

"Il se dissimulait dans les fissures du sol et attendait, béatifié, que quelques eaux le germe" ${ }^{125}$.

Le Clézio écrit aussi dans L'Inconnus sur la terre :
"Je ressens le désir du réel(...) le savoir non avec son intelligence, mais avec ses sens, avec sa vie(...) J'ai faim et soif de chaleur, de vent, de pluie, de lumière(...) je veux toucher la terre, la sentir, je veux être tout entier en elle"

Pareillement, le froid, l'ombre l'eau qui représentent les éléments négatifs de Yin pénètrent dans leurs contraires. Dans $\underline{\text { L'Inconnu }}$ sur la terre, Le Clézio célèbre les éléments de la nature et fait de 
son œuvre quelque chose qui s'approche de la prose élevée. En plus, il associe les éléments de vie et de mort, le clair et l'obscur, le chaud et le froid, le masculin et le féminin. Cela est très sensible dans les passages de son œuvre intitulée L'extase $\underline{\text { matérielle }}$ où se montre cette interprétation mutuelle des éléments de Yin et de Yang ${ }^{27}$.

En réalité, Le Clézio excelle à peindre en images vivantes cette harmonie sensuelle et cosmogonique entre les éléments de la nature. Cela est visible dans son ouvrage $\underline{H a}^{28}$ où il essaie de réconcilier la présence des forces opposées afin de former une unité à partir du contraste. Dans $\underline{\text { Terra Amata }}$, la société humaine et les cités sont décrites de cette façon :

\section{"l’enfer et le paradis réunis sur le même lieu, et on ne peut habiter l'un sans habiter l'autre" ${ }^{29}$.}

C'est ainsi que les contradictions irréconciliables soustendent la pensée et la philosophie de Le Clézio dans sa quête de la vérité :

\section{"Les deux se mêlent, surgissent ensemble, résonnent en moi comme une seule et même émotion" ${ }^{\prime 30}$.}

Ajoutons que Le Clézio reconnaît que le langage humain reste encore incapable d'exprimer l'infinité des sentiments et les 
nuances émotionnelles qui se mêlent, se confondent et changent toujours dans l'homme. Pour rapprocher cette notion, Le Clézio donne exemple qui simplifie cette question:

\section{"peut-être est-ce en jetant les contrastes qu'on obtient le mélange de la vie ; gris = noir + blanc"31.}

Mais il reprend très vite et dit :

\section{"Même pas. Enchevêtrement des milliards de couleurs, dont chaque nuance ne sert pas deux fois" ${ }^{\prime 32}$.}

Catégoriquement, Le Clézio refuse la dichotomie entre les antithèses comme la vie et la mort, le réel et l'imaginaire. Pour lui, la force de l'art est de révéler et de mettre en relief les contrastes et les oppositions ensemble pour faire la jonction de l'imaginaire et du réel, de l'illusion et de la réalité.

En effet, le dénominateur commun dans les ouvrages de Le Clézio s'incarne dans sa recherche d'une esthétique nouvelle procédant de la fusion mutuelle des antithèses. Par conséquent, le réel existe avec le fictif en interpénétration mutuelle, mais dans la mesure où il conduit à une compréhension profonde de l'existence.

Dans Le livre des fuites surtout, Le Clézio cherche à fusionner ensemble le réel et l'imaginaire en utilisant des modes 
variés comme la poésie, le discours scientifique objectif, le journal de voyage, la critique littéraire. Dans cette perspective, Le Clézio se projette dans ce livre pour coexister à côté de ses personnages fictifs. Parfois, il alterne avec eux à travers le jeu de la première et de la troisième personne. Quelquefois aussi, Le Clézio annonce implicitement sa présence pour souligner la nature fictive de ses créations imaginaires :

\section{"Assez de je ! C'est lui, c'est moi devenu ami, dont je veux parler" ${ }^{33}$.}

Bref, il n'y a presque pas de distance qui sépare le monde fictif du monde réel chez Le Clézio. Le monde réel, comme le monde imaginaire, se dérive des arrangements possibles et variés et des réarrangements des éléments inspirés et fournis par la réalité extérieure.

D'ailleurs, Le Clézio voit que le monde est une entité vivante. Telle est la vision centrale sur laquelle débouche son œuvre. Un arbre, un caillou, une goutte d'eau ont une vie et le monde entier se bat et se propulse. Tout objet a une vie particulière si l'on voit et examine bien les choses. C'est ainsi que l'œuvre de cet écrivain est dominée par un certain regard qui dissèque et pulvérise les choses. Par conséquent, Le Clézio pense qu'on peut saisir le monde dans son combat insatiable par un exercice inlassable de l'imagination ${ }^{34}$. 
Disons encore que dans l'immobilité apparente du corps, le personnage chez Le Clézio, comme lui-même, perçoit les êtres et les choses qu'il contemple. Il saisit chaque geste, chaque sensation et sent en lui-même la présence du monde hors des limites spatio-temporelles. C'est ainsi qu'il peut fuir le monde prochain et glisser d'une forme à une autre forme. C'est pourquoi, dans son ensemble, l'œuvre de Le Clézio ressortit, à côté du thème de la fuite, la quête de ce qu'il peut appeler : la conscience ou la pensée.

Tous ses personnages incarnent le même désir partout et pareillement à travers la fuite et le déplacement dans l'espace et dans le temps. Mais le problème réside dans l'impossibilité de mettre une fin à ce mouvement perpétuel pour l'accomplissement du même désir :

"Ce désir c'est celui de l'immobilité, du repos que les stridences de la vie moderne refusent à l'homme" ${ }^{35}$.

C'est ainsi que le mal dont souffre Le Clézio se concrétise, selon lui, dans l'esclavage d'une civilisation dominée par des valeurs purement matérielles. Pour lui "la civilisation et la modernité troublent la vie de l'homme en lui donnant du stress et des perturbations ${ }^{136}$. D'après lui, la solution réside dans le fait de changer la civilisation ${ }^{37}$. Nous pouvons donc dire que ses premiers ouvrages révèlent, pour ainsi dire, l'enfer du monde 
moderne et les méfaits de la civilisation. Il attaque ce monde à travers les mots et les images. Il dénonce tout ce qui est inhumain dans cette civilisation et invite ses lecteurs à fuir à tout prix de cet emprisonnement et à se libérer "des pièges que des Maîtres tout puissants ont déposés devant chacun de nos pas, de nos moments, de nos pensées ${ }^{\prime 38}$.

En fait, Le Clézio cherche à se libérer de l'asservissement des maîtres et de leurs machines et même de leur langage par la vraie pensée de la communication immédiate entre l'individu et le monde. Cependant, il semble que Le Clézio échappe du monde mécanique et ses objets pour tomber dans le règne de la matière et du mutisme. Il est donc constant de trouver chez Le Clézio un rapport spécifique "aux objets qui est de type haine-amour. À force de haïr les choses, on finit par envier leur force silencieuse, leur automne muet ${ }^{\prime 39}$.

Malgré tout, Le Clézio découvre, dans la ville qu'il dénonce, des objets à aimer comme les moteurs, les voitures, les lumières et surtout le néon :

"quelquefois même, on sent de la haine devant ces moteurs, mais c'est comme cela qu'on commence à les aimer parce qu'ils ne renvoient leur haine vers vous. Les machines ne trahissent pas" ${ }^{\prime 40}$. 
C'est dans cette optique que se précise la vision que Le Clézio porte sur le monde moderne, ses objets et ses matières. Pour éclairer ses angoisses et ses sentiments contradictoires, pour ainsi dire, Le Clézio présente des personnages imprégnés par une vision qui fonction, comme dit Fabre-Luce Anne, d'après la "double fascination pour les forces du chaud et du froid qui reparaît dans ses romans : celle du soleil de l'Icarisme fatal (le chaud, et celle de la minéralisation (le froid) "A1. Autrement dit, la pensée et la philosophie de Le Clézio se fondent sur cette vision minéralisée ou motrice des objets, et sur un regard qui occulte l'énergie vitale de ces objets ${ }^{42}$.

Nous pouvons donc considérer la pensée de Le Clézio comme une recherche sur la nature du monde et celle de l'homme sur la réalité d'une mesure éthique de la réalité qui est la vérité.

Enfin, nous pouvons dire que la pensée de Le Clézio n'est pas fondée sur des concepts rationnels qui doivent aboutir à une interprétation logique, mais sur le moyen d'une expression métaphorique qui correspond à une vision grandiose et profonde d'une prise de conscience sans limites.

Par conséquent, la réalité dans ses manifestations à l'intérieur du cadre du temps et de l'espace, et la conscience, miroir de l'homme dans la quête du soi et du monde, sont les 
deux pôles principaux qui sous-tendent la méditation philosophique de Le Clézio.

- La recherche de soi et d'un nouveau monde :-

Le Clézio s'évertue à franchir la réalité du monde extérieur vers la vérité et les secrets du monde intérieur en vue d'approfondir son expérience et sa vision du monde. Son œuvre révèle plutôt une étendue psychique comme un monde doué de vie et de mouvement :

"La psychanalyse est arrivée au moment propice pour répondre à cette angoisse uni verselle : elle a permis à tout un chacun de se délivrer de ses démons en prônant une réconciliation avec soi-même(...). La seule solution réaliser une véritable recherche identitaire resterait donc la fuite dans un monde qui ne soit pas industrialisé, un monde où l'exilé pui sse enfin se réaliser par une quête de soi sans être conditionné par un système répressif ${ }^{0433}$.

La création littéraire et l'emploi des termes d'un espace qui renvoie tantôt au monde extérieur tantôt au monde intérieur sont des supports susceptibles à la symbolisation du moi et à sa définition face à d'autres sujets ${ }^{44}$.

C'est ainsi que l'écrivain ne cesse de refléter à la fois cette expérience sensorielle et affective à travers son œuvre. Parmi les

(La Fuite et la recherche de soi...) Dr. Noha Abdel Aziz Rizq 
expressions récurrentes que l'œuvre propose, celle en est une de plus incessante inscrite dans son contenu : recherche de l'inconnu, errance physique et psychologique, quête de soi et du monde :

\section{"La conscience c'est le moi projeté sur le monde, miroir qui renvoie l'image ${ }^{\prime 45}$.}

Par conséquent, Le Clézio et ses personnages confrontent une dualité de sens, comme la vie et la mort, la réalité et l'illusion pour arriver à la certitude et à la vérité de soi et du cosmos. Le monde extérieur et le monde intérieur sont donc conçus à travers une imagination féconde et créatrice.

L'originalité de Le Clézio réside dans le fait de faire sentir la présence même du monde dans ses ouvrages et de "nous y immerger vivants, tout pantelants, avec les précisions, les images d'un styliste doublé d'un dramaturge et d'un poète, car c'est par le ressassement, l'obsession, le harcèlement, par de variation sur le même thème, la place du moi dans l'universelle création qu'il essaie d'y parvenir". 46

On doit remarquer que Le Clézio situe son personnage comme un être vivant parmi des millions des êtres vivants, sa pensée tend vers la connaissance de l'homme plongé dans l'univers où il est condamné à vivre et à mourir. D'autre part, Le Clézio reconnaît que l'intelligence humaine ne peut saisir l'inintelligible, car l'univers est enfermé sur son propre secret. 
Cependant, Le Clézio opte pour une relation d'échange et d'harmonie entre l'homme et le cosmos.

Nous pouvons ajouter que le personnage chez Le Clézio est un être libre qui pourrait sembler, de prime abord, sans aucun engagement qui empêche de méditer, d'errer ou de voyager. Il se laisse donc aller dans l'univers pour goûter à tout plaisir et savourer à toute sensation sachant la valeur du temps et la préciosité de la vie où "le présent est triomphant, bâti sur les deux infinis, dont l'un s'en va et l'autre arrive't7.

Le Clézio veut ainsi saisir le mieux possible la réalité du présent où il vit. C'est pourquoi il dit dans l'extase matérielle :

"Je vois, j’entends, je sens. Je goûte, je ressens. Il y a sûrement un moyen de retrouver le chemin du monde à travers mon corps". 48

Il est important de signaler aussi que l'univers romanesque de Le Clézio est un univers enfantin pour ainsi dire, car l'enfant est un être privilégié par son ouverture sur le monde et son regard neuf vers la vie et les choses. Cet enfant dans l'œuvre de Le Clézio a de fortes relations avec l'univers et ses secrets. C'est pourquoi Le Clézio choisit, souvent, ses personnages parmi les enfants. On le trouve dans les nouvelles intitulées Mondo et $\underline{\text { autres histoires }}^{49}$, l'enfant Mondo qui vit sur la terre comme un roi et le petit garçon inconnu dans l'inconnu sur la terre, ou 
encore Hélène dans $\underline{\text { Étoile errante }}{ }^{50}$ qui ne quitte ni les souvenirs de son enfance ni sa relation avec sa patrie à travers ses voyages dans la vie.

Il s'agit donc dans ses récits romanesques d'errance et de voyages, de nostalgie et de souvenirs à travers l'espace et la mémoire qui a pour fonction d'écourter la distance qui sépare le passé du présent et de réactualiser l'expérience du passé. Véronique Giorigiutti considère l'écriture de Le Clézio est une des causes grâce auxquelles le lecteur se sent libre où il dépasse toutes les entraves et toutes les limites de l'espace et du temps : "Peu d'écritures me sont aussi libératrices que celle-ci, qui n'est encore qu'au milieu d'elle, qui part sans cesse du milieu, qui n'est ni moraliste ni craintive, ni arrangée, ni difficile ni facile, extrême contradiction, pratique et critique, romanesque et cosmopolitique $^{151}$.

En effet, l'œuvre de Le Clézio représente quelque parallélisme entre le présent et le passé idéalisant ainsi les valeurs humaines perdues par l'écart du temps. Cette comparaison permet au personnage, dans les romans de Le Clézio, de découvrir le monde et de poursuivre son voyage dans la vie.

Notons aussi, dans ses ouvrages, que le temps est symbolisé par un retour à l'origine, par la remontée d'un passé lointain ou immémorial. L'intemporel y règne pour permettre aux 
désirs et aux rêves de jaillir au sein de l'espace du récit romanesque.

En effet, Le Clézio insiste sur le fait de saisir et d'interpréter les mouvements imperceptibles des sentiments en un langage susceptible de situer le moi dans l'ensemble du cosmos. Il s'intéresse à l'entreprise existentielle et à la prise de contact avec les forces qui animent ce cosmos. Le Clézio traduit cela par le sentiment qu'il a de vivre dans le monde, par l'envahissement incessant des sensations externes et des sentiments internes comme la joie, la douleur, l'attente, la peur et l'espoir. Le Clézio s'avère saisir, par la crainte et la contrainte, tout hasard, s'adapter à toutes les circonstances de sa vie et de l'existence même. Cette idée est appuyée par Maurice Nadeau dans son livre sur le roman français depuis la guerre ${ }^{52}$.

Quand on lit l'œuvre de Le Clézio, on découvre que ses phrases sont parfois rapides et quelquefois haletantes. Cela pourrait expliquer sa grande vision du monde puisqu'il veut embrasser les diverses manifestations cosmiques et les sentiments humains à travers ce qu'il écrit. Sa vision est donc une vision ouverte et va directement avec le monde dans lequel il est plongé. Il voit en même temps, le monde avec les yeux d'un poète sensible et ceux d'un philosophe lucide. En lisant ses écrits, on découvre qu'il insiste sur la création permanente d'un langage 
capable de traduire et de peindre les images du monde en mots. Il dit :

"Je voudrais vous parler loin. Longtemps, avec des mots qui ne seraient pas seulement des mots, mais qui conduiraient jusqu'au ciel, jusqu'à l'espace, jusqu'à la mer"153.

C'est ainsi que Le Clézio s'efforce de recréer la beauté du monde à travers les mots qui cristallisent les idées et incarne le monde en images vivantes. Dans L'inconnu sur la terre, Le Clézio essaie de faire de la musique par les mots, voyager et vivre, voir et rêver, écrire pour une nouvelle vie. Il est convaincu que c'est la parole du poète ou de l'écrivain qui doit recréer les choses, faire sentir cette harmonie entre l'homme et l'univers. Le Clézio explique, dans l'Essai déjà cité, ce que désigne le langage des signes. Il dit :

"Je voudrais qu'il n'y ait pas de différence entre les éléments et les hommes, entre la terre, le ciel, la mer et les hommes. Je voudrais pouvoir comprendre les signes, au même moment, les entendre au même moment. Je voudrais qu'il n'y ait qu'un seul sentiment qui irait sans cesse de la terre vers les hommes et retournerait vers la terre comme un courant, comme un mouvement de flux et de reflux" ${ }^{\prime 54}$. 
Comme on vient de le montrer, Le Clézio exprime une tendance d'animation et d'identification du tout en tout par un mélange de l'imaginaire et du réel. D'après lui, écrire, c'est donna à l'abstrait un souffle de vie et de mouvement. L'expérience du réel se reflète dans toute sa charge, sa vivacité et son ampleur à travers l'espace du texte écrit. Les deux espaces s'approchent et se rejoignent :

\section{"Je voudrais qu'il n'y qu'un seul langage (...) Je vois l'espace, je suis dans l'espace. Ce qui sépare les hommes du monde du réel va disparaître (...) enfin le monde va venir, va se répandre, s'unir. Tout va parler ...." ${ }^{55}$}

Remarquons, d'autre part, que la pensée de Le Clézio a quelque chose de particulier : parvenir à libérer le langage de tout ce qui l'entrave ou le trouble. Le langage doit être l'interprète du silence, de la lumière, du ciel, de la lumière, du ciel, de la mer... etc. ${ }^{56}$ Le Clézio voit le monde avec le regard d'un poète sensible et d'un peintre habile. On pouvait dire ainsi que l'intention de Le Clézio et sa conception de la vie consistent à révéler un moi dans un monde plein de contradiction et de changements. Mais cette conception de Le Clézio est à la fois assez claire et assez obscure surtout lorsqu'il montre le moyen de cerner ce qui se passe dans le monde extérieur; d'après lui c'est de révéler le conflit intérieur que représente le moi. La philosophie de cet écrivain se fonde sur la présence des idées et des concepts contradictoires qui 
combinent leur force, aucune pour soi, aucune pour l'autre, mais tous pour tous :

\section{"La littérature atteint parfois ce bouleversement, elle nous apporte une compréhension, une émotion très forte qui nous guérit souvent de nous-mêmes, qui nous porte en tous cas vers les autres et nous aide à vivre ensemble $e^{1157}$.}

Pour Le Clézio, renoncer à la contradiction est impossible, car ce serait renoncer à la vie elle-même.

D'autre part, l'écrivain prouve sa vision à travers une comparaison du monde intérieur et du monde extérieur, du monde réel et du monde imaginaire, et ainsi d'une série interminable de sens opposés.

Dans le livre des fuites, le narrateur s'identifie au personnage qui refuse le concept de l'identité pour être «un» ou d'avoir même un nom unique. Le personnage du livre devient parfois homme, puis femme, puis homme encore. Dans son aventure à travers le monde, il prend plusieurs prénoms: Jeune Homme Hogan ou simplement J.H.H., Jeune Hombre Hogan, Junatio Holgzan ... Ce personnage semble être dispersé et perdu dans la foule, contraire à lui-même et à son intégrité individuelle. Il perd aussi l'authenticité ou la vraisemblance d'être localisé dans l'espace et dans le temps : 
"On pourrait s'appeler Hogan, aussi, et être un homme de race blanche. Né à Langson (Vietnam) il y avait à peu près vingt-neuf, trente ans. Habitant un pays qui s'appelait La France, parlant, pensant, rêvant, désirant en une langue qui s'appelait le français. Et c'était important : si on s'était appelé Kamol, né à Chantanaburi ou bien Jésus Torre, né à Sotolito, on aurait eu d'autres mots, d'autres idées, d'autres rêves ... J'aurais pu commencer ceci de mille façons différentes" ${ }^{1058}$.

Ajoutons que l'écriture même chez Le Clézio peut subir une constante modification et se varie. Un mot entraine un autre mot et un concept contredit un autre concept. Mais chez Le Clézio, les concepts se contredisent pour s'affirmer et se concrétiser. C'est ainsi que nous remarquons un état permanent de présence - absence, d'affirmation - négation et de synchronie diachronie. Cela peut faire croire que Le Clézio est un romancier antiformaliste qui défie les formes et les règles convenues ${ }^{59}$.

Comme nous l'avions déjà signalé, Le Clézio dénonce certains aspects de la vie, de la pensée et de la culture occidentale, sachant, en même temps, qu'il ne peut pas les rejeter totalement puisqu'il a été formé par elles. 
D'ailleurs, pour Le Clézio la compréhension de soi et de la conscience individuelle des faits ne se sépare pas de la compréhension du monde extérieur. La responsabilité de l'homme est donc de découvrir sa place et d'assumer son rôle dans le monde.

\section{"Il peint des êtres humains qui fuient la ville, espace inhumain, ou la civilisation pour communier avec les éléments naturels ou communiquer avec l'invisible et vivent des expériences de fusion avec le cosmos, ou d'union avec l'univers. C'est alors qu'opère la magie de l'écriture le clézienne. L'artiste rejoint l'homme dans sa quête d'harmoni $\mathrm{e}^{\text {"60 }}$.}

Dans cette quête Le Clézio se sert d'un style composé de l'illusion et de la réalité, de l'affirmation et de la négation, de l'extérieur et de l'intérieur, de la superficie et de la profondeur en vue d'aboutir à une connaissance plus sûre de sa conscience et de son existence même dans le monde. Dans le chapitre sur le silence dans $\underline{\text { l'extase matérielle, }}{ }^{61}$ Le Clézio reconnaît qu'il n'y a pas moyen d'échapper au langage puisqu'il est une chose déterminée et inéluctable. Mais les chances de découvrir la vérité à travers le langage sont minimes. C'est pourquoi il donne la préférence au silence et à la méditation profonde. 
Par rapport au monde, Le Clézio pense que l'intelligence et les systèmes de connaissance ne sont que des instruments de doute, de séparation et d'abstraction. La conscience individuelle semble être bourrée et incapable d'appréhender tout acte comme procès unique. C'est pourquoi Le Clézio établit toujours avec le monde des relations directes d'osmos et d'interaction et d'interprétation pour arriver à une meilleure compréhension de soi et de l'univers. Son œuvre révèle plutôt un principe psychologique basé sur la conscience assimilée à un miroir ou à la vue qui revient sur elle-même.

D’après les œuvres de Le Clézio qui forment le corpus de cette étude, on pourrait dire, à juste titre que la fuite de la ville et le refus du milieu citadin causeraient un certain malaise et une espèce d'inadaptation entre l'individu et le milieu où il habite et vit. Cela entraîne une rêverie ou un désir inconscient de rêver sans aucun lien avec la réalité. C'est ainsi que le rêve et le refus de la réalité s'imbriquent sans qu'il soit possible de discerner la logique de telles aspirations, puisque le réel est absent et banni parce qu'il ne correspond pas au souhait initial. La fuite dans le rêve est une sorte de compensation à travers laquelle l'individu peut se mouvoir dans un espace sans entraves. Le vol dans l'espace est donc un procédé récurrent dans l'œuvre de Le Clézio. Il s'agit de planer dans la vacuité et l'immensité de la sphère céleste. Comme 
dans le rêve, ce désir représente une sorte de détachement inconscient du monde terrestre et ses problèmes.

En effet, Le Clézio montre dans ses ouvrages que le rêveur éprouve une joie en planant à travers les régions spacieuses. Cela se trouve explicitement dans l'inconnu sur la terre ${ }^{62}$. Cette sensation est confirmée par Mathe (Roger) dans son livre sur l'exotisme d'Homère à Le Clézio lorsqu'il dit :

\section{"Chaque être se sent prisonnier du cercle où le hasard le fait vivre" 63 .}

La fuite, le voyage et le déplacement constituent un remède qui peut vaincre la lassitude et calmer l'angoisse. Cette fuite transmet l'individu du cercle étroit qui l'entoure et le rend capable de surmonter le milieu matériel ou même spirituel ou il est astreint à vivre. Pour évoquer des souvenirs et retourner au passé, il faut une errance à travers l'espace et le temps afin de restituer les indices et les traces évanouies dans le mémoire.

Par conséquent, nous avons voulu prouver dans cette modeste étude sur la fuite dans l'œuvre de Le Clézio, que le personnage dans les différents écrits de cet auteur se démarre dans sa quête de soi et du monde sur deux plans : un plan réel et un autre plan imaginaire. Plus son présent l'accable, plus il est tenté de fuir, de s'évader et de s'éloigner. 
Le personnage, chez Le Clézio, incarne la nostalgie du retour au passé et a une vie simple ; et tout cela dans la recherche de l'inconnu, du lointain dans l'espace et dans le temps. Un tel personnage a un besoin de départ et cherche une ouverture sur un nouveau monde et un retour à une vie plus simple et moins compliquée.

Disons enfin que la fuite chez Le Clézio permet de passer à la vraie recherche de soi et même d'un nouveau monde en essayant d'abolir les limites. C'est un monde transparent et limpide que seule la fuite permet d'y arriver. 


\section{$\underline{\text { Marge }}$}

(1) Arouna (Coulibaly), "De l'évasion à l'itinérance: la mobilité comme refuge dans le livre des fuites de Jean-Marie Gustave Le Clézio", in Recherches africaines, annales de l'université des lettres et sciences humaines de Bamako, nº 21, juin 2018, P.107.

(2) Le Clézio (J.M.G), "Le livre des fuites", Paris, Gallimard, 1969, P. 88.

(3) Kadioğlu (Sevket), "J.M.G Le Clézio : refus de l'Eurocentrisme et l'appel de l'ailleurs" in Journal of Faculty of lettres cift 1 vol. 24, $\mathrm{n}^{\circ}$ 2, Décembre 2007, PP. 131, 132.

(4) Le Clézio (J.M.G), "Terra amata", Paris, Gallimard, 1967, P. 28.

(5) Kadioğlu (Sevket), "J.M.G Le Clézio : refus de l'Eurocentrisme et l'appel de l'ailleurs", op.cit., P. 132.

(6) Ibid., P. 129.

(7) Ségadie (Ilham), Faroghie (Hassan), "Retour au désert ou refus de la modernité dans Désert de Le Clézio", Téhéran Université Azad islamique Téhéran, Unité de Sciences et de Recherches, Doctorante, in Revue des Etudes de la langue française, article _ 20286 < relf.ui.ac.ir., P. 26.

(8) Hanquier (Eddy), "Parole et silence chez Le Clézio", in communication \& langages, année 1991, P. 18.

(9) Mohamed (El Bouazzaoui), "Modalités de la fuite et de l'errance dans quelques textes de Jean- Marie Le Clézio", in voyage, errance et exil. Parcours existentiels. [lisbn:9789954379165], 2016, P. 43.

(10) Le Clézio (J.M.G.), "Le livre des fuites", PP. 86, 87.

(11) Ibid., P. 140. 
(12) Ibid., P. 90.

(13) Ibid., P. 88.

(14) Ibid., P. 89.

(15) Ibid., P. 88.

(16) Ibidem.

(17) Mohamed (El Bouazzaoui), "Modalités de la fuite et de l'errance dans quelques textes de Jean- Marie Le Clézio", op.cit., P. 71.

(18) Thibault (Bruno), "Le livre des fuites de J.M.G. Le Clézio et le problème du roman exotique moderne", in The French Review, vol. 65, No. 3, Feb. 1992, P. 427.

(19) Le Clézio (J.M.G.), "Le livre des fuites", P. 175.

(20) Ibid., P. 108.

(21) Cagnon (Maurice) et Smith (Stephen),"Le Clézio Taö̈ste vision" in The French Review, n ${ }^{0}$ 6., 1974, PP. 245, 252.

(22) Taôisme ou Taoïsme est une religion populaire d'extrême Orient fondée au $6^{\mathrm{e}}$ siècle avant J.C par le chinois Lao-Tseu.

(23) Le Clézio (J.M.G.), "Terra Amata", P. 229.

(24) Le Clézio (J.M.G.), "Le Procès-Verbal", Paris, Gallimard, 1963, PP. $69-70$.

(25) Ibid., P. 77.

(26) Le Clézio (J.M.G.), "L'inconnu sur la terre", Paris, Gallimard, 1978, P. 158.

(27) Le Clézio (J.M.G.), " L’extase matérielle ", Paris, Gallimard, 1967, PP. 123, 124.

(28) Le Clézio (J.M.G.), " Haï ", Paris, Gallimard, 1971, PP. 94 - 95.

(29) Le Clézio (J.M.G.), "Terra Amata", P. 238. 
(30) Le Clézio (J.M.G.), "L’extase matérielle", P. 123.

(31) Ibidem.

(32) Ibidem.

(33) Le Clézio (J.M.G.), "Le livre des fuites", P. 186.

(34) Voir Le Clézio (J.M.G.), "L’extase matérielle", PP. 229 - 250.

(35) Voir Fabre-Luce (Anne), "La fuite immobile", dans la quinzaine littéraire $n^{0} 73$, P.6.

(36) Ségadie (Ilham), Faraghie (Hassan), "Retour au désert où refus de la modernité dans Désert de Le Clézio", op.cit., P. 25.

(37) Voir Bott (François) dans son entretien avec Le Clézio, Le Monde, 12 avril, 1973, P.20.

(38) Voir Fabre-Luce (Anne), "L'enfer de la matière : J.M.G. Le Clézio", dans la quinzaine littéraire $\mathrm{n}^{\mathrm{o}} 163,1973$, P.7.

(39) Ibidem.

(40) Ibid., P. 8.

(41) Ibidem.

(42) Ibidem.

(43) Thérond (Emmanuel), "Exile et quête de soi chez J.M.G. Le Clézio (voyage à Rodrigues), Paulo Coelho (L'Alchimiste) et Rachid Boudjedra (Timimoun) ", Université Blaisé Pascal Clermont Fernand II, Maîtrise de lettres modernes, 2001, P. 6.

(44) C.F. Tadié (Jean. Yves), "Le récit poétique", Paris, P.U.F., 1978, P. 51.

(45) Di Scanno (Teresa), "La vision du monde de Le Clézio", Paris, Nizet, 1983, P.17.

(46) Ibidem.

(47) Le Clézio (J.M.G.), "L’extase matérielle", P. 145. 
(48) Ibidem.

(49) C.F. Le Clézio (J.M.G.), "Mondo et autres histoires", Paris, Gallimard, 1978.

(50) C.F. Le Clézio (J.M.G.), "Étoile errante", Paris, Gallimard, 1992.

(51) Giorigiutti (Véronique), "Migrations de J.M.G. Le Clézio", in Ecologie \& Politique, N $N^{0}$ 36, Feb. 2008, Pages 41 à 51.

(52) Nadeau (Maurice), "Le Roman français depuis la guerre", Paris, Gallimard, 1970, P. 219.

(53) Le Clézio (J.M.G.), "L’inconnu sur la terre", P. 7.

(54) Ibid., P. 116.

(55) Ibid., P. 161.

(56) C.F. Salgas (Jean-Pierre), "J.M.G. Le Clézio : "lire, c’est s'aventurer dans l'autre" dans Entretien 1983 - 1990, 17 minutes de lecture in Quinzaine littéraire $n^{\circ} 435,1^{\text {er }}$ mars 1985.

(57) Roussel-Gillet (Isabelle), "En dialogue avec Jean-Marie Gustave Le Clézio, sous le signe de la fantaisie", dans Roman 20 50, 1/2013, nº 55, PP. $131-146$.

(58) Le Clézio (J.M.G.), "Le livre des fuites", PP. 23 - 24.

(59) Ibid., PP. $54-55$.

(60) Bolle (Georges), "Le Clézio et la quête d'harmonie", in Astrolabe $\mathrm{N}^{\mathrm{o}} 46$, Novembre/ décembre 2014.

(61) Le Clézio (J.M.G.), "L'extase matérielle", Voir le chapitre sur le Silence de P.263 à P. 315.

(62) Le Clézio (J.M.G.), "L'inconnu sur la terre", voir P. 208 et les suivantes. 
(63) Mathe (Roger) "L'exotisme d'Homère à Le Clézio", Paris, Bordas, 1972, P. 14. 


\section{Bibliographie}

\section{I- Corpus :-}

Le Clézio (J.M.G),

- $\quad$ "Procès-verbal", Paris, Gallimard, 1963.

- $\quad$ "L’extase matérielle", Paris, Gallimard, 1967.

- $\quad$ "Terra Amata", Paris, Gallimard, 1967.

- $\quad$ "Le livre des fuites", Paris, Gallimard, 1969.

- "Haï", Paris, Sentiers de la création, 1971.

- $\quad$ "L’inconnu sur la terre", Paris, Gallimard, 1978.

- $\quad$ "Mondo et autres histoires", Paris, Gallimard, 1978.

- "Étoile Errante", Paris, Gallimard, 1992.

\section{II- Ouvrages généraux :-}

- Nadeau (Maurice), "Le Roman français depuis la guerre", Paris, Gallimard, 1970.

- Tadié (Jean. Yves), "Le récit poétique", Paris, P.U.F., 1978.

\section{III- Ouvrages critiques consacrés à Le Clézio :-}

- Di Scanno (Teresa), "La vision du monde de Le Clézio", Paris, Nizet, 1983.

- Mathe (Roger) "L'exotisme d'Homère à Le Clézio", Paris, Bordas, 1972.

\section{IV- Articles sur Le Clézio :-}

- Arouna (Coulibaly), "De l'évasion à l'itinérance: la mobilité comme refuge dans le livre des fuites de Jean-Marie Gustave 
Le Clézio", in Recherches africaines, annales de l'université des lettres et sciences humaines de Bamako, $n^{0}$ 21, juin 2018.

- Bolle (Georges), "Le Clézio et la quête d'harmonie", in Astrolabe No. 46, Novembre/ décembre 2014.

-Bott (François) dans son entretien avec Le Clézio, Le Monde, 12 avril, 1973.

- Congnon (Maurice) et Smith (Stephen)'Le Clézio Taoïste vision" in The French Review, Nº 6, 1974.

- Fabre-Luce (Anne), "L’enfer de la matière : J.M.G. Le Clézio", dans la quinzaine littéraire no 763, 1973.

•Giorigiutti (Véronique), "Migrations de J.M.G. Le Clézio", in Ecologie \& Politique, No. 36, Feb. 2008.

- Hanquier (Eddy), "Parole et silence chez Le Clézio", in communication \& langages, année 1991.

-Kadioğlu (Sevket), "J.M.G Le Clézio : refus de l'Eurocentrisme et l'appel de l'ailleurs" in Journal of Faculty of lettres cift 1 vol. 24, no 2, Décembre 2007.

- Mohamed (El Bouazzaoui), "Modalités de la fuite et de l'errance dans quelques textes de Jean- Marie Le Clézio", in voyage, errance et exil. Parcours existentiels, (lisbn:9789954379165).

-Roussel-Gillet (Isabelle), "En dialogue avec Jean-Marie Gustave Le Clézio, sous le signe de la fantaisie", dans Roman 20 - 50, 1/2013, no 55 . 
-Salgas (Jean-Pierre), "J.M.G. Le Clézio : "lire, c'est s'aventurer dans l'autre" dans Entretien 1983 - 1990, 17 minutes de lecture in Quinzaine littéraire no 435, 1er mars 1985.

- Thibault (Bruno), "Le livre des fuites de J.M.G. Le Clézio et le problème du roman exotique moderne", in The French Review, vol. 65, No. 3, Feb. 1992.

\section{V-Thèses :-}

-Ségadie (Ilham), Faroghie (Hassan), "Retour au désert ou refus de la modernité dans Désert de Le Clézio", Téhéran Université Azad islamique Téhéran, Unité de Sciences et de Recherches, Doctorante, in Revue des Etudes de la langue française, article _ $20286>$ relf.ui.ac.ir.

-Thérond (Emmanuel), 'Exile et quête de soi chez J.M.G. Le Clézio (voyage à Rodrigues), Paulo Coelho (L'Alchimiste) et Rachid Boudjedra (Timimoun) ", Université Blaisé Pascal Clermont Fernand II, Maîtrise de lettres modernes, 2001. 


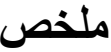

موضوع الهروب له أهية كبري، ويحتل مكاناً أساسياً في أعمال الروائي لوكليزيو بداية من روايته الأولي. وفي جميع رواياته وخاصة رواية "كتاب الهروب" والذي لا يستعرض فيه الكاتب إلا تفاقم القلق من العيش في وئام في هذا العالم المادي الحديث (الحياة في المدن)، لدرجة أنه يريد الهروب والفرار دائماً ومغادرة المكان والزمان، والبحث عن ذاته في الطبيعة. وقد يكون هذا الهروب هروباً معنوياً بالدرجة الأولي، وغالباً ما يصحبه هروبا حقيقياً بالمعني الدقيق للكلمة. وبالنظر إلي أعماله، نجد أن حياته وعمله بعتمدان علي نقطتين جوهريتين : الإنجذاب إلي حياة المدينة من جهة، والإرتباط بالطبيعة وعناصرها من جهة أخري. ويؤكد لوكليزيو دائماً أن الحياة في المدينة وجميع القيم المادية قد غيرت الصورة الجميلة للأرض، لدرجة أنها أفسدت القيم الإنسانية. فهو مقتنع تماماً أن اللغة التي تهيمن علي الحياة الحديثة لم تعد اللغة التي تسعي إلي الترابط والسلام والمحبة والتعاون بين أفراد المجتمع، بل أنها أصبحت اللغة التي تقوم علي المصلحة الخاصة، والأكاذيب، والنفعية، والهيمنة التي تصل إلي العبودية بين أفراد المجتمع. وهذا هو سبب قلق وعدم ثقة لوكليزيو في هذا العالم الحديث. وتبرز رواية "كتاب الهروب" تصاعد العنف والوحدة، والخوف وبالتالي الهروب الذي يشكل موضوع هذه الدراسة حيث يكون للهروب جانبان أساسيان: الهروب من الحياة المادية الحديثة والبحث عن الذات وعالم جديد. 